\title{
A Detailed Kinematical Study of the Point-Symmetric Planetary Nebula IC4634
}

\author{
O. Toledano*, H. Riesgo-Tirado*, J.A. Lopez*, J. Meaburn**, M.

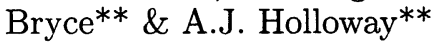 \\ *Instituto de Astronomía UNAM-Ensenada Apdo. Postal 877, \\ Ensenada, B.C. 2800, México \\ ** Jodrell Bank Observatory, Univ. of Manchester.
}

\begin{abstract}
IC 4634 is a planetary nebula with a striking point-symmetric morphology. Strings of line-emitting knots, particularly bright in [N II] emerge from its bright $\mathrm{H} \alpha$ core. These strings bend in opposite directions on either side of the nucleus. We have obatined sub-arcsecond monochromatic imagery that neatly reveals its main morphological components. Additionally, we also present a comprehensive kinematic mapping of the nebula, consisting of several long-slit echelle positions across the main components of the nebula.
\end{abstract}

The long-slit, echelle spectra and images were obtained under unusually good seeing conditions (FWHM $=0.7$ arcsec) on two separate runs, from the southern and northern hemisphere. The Manchester Echelle Spectrometer (Meaburn et al, 1984) was used at the Anglo-Australian Telescope and its twin brother at the San Pedro Martir Observatory. The [N II] emission conspicuously highlights the string of point-symmetric knots. The $\mathrm{H} \alpha$ emission contributes mainly to the extended, elliptical envelope whereas the [S II] emission is particularly prominet at the core in a bar-like structure. Line profiles show blueshifted and redshifted components on both sides of the core with respect to the systemic velocity. This kinematic behavior is indicative of bipolar, episodic ejecta ( e.g. Lopez 1997). A velocity ellipse has been detected for the first time at the center of the IC 4634, revealing the presence of an inner expanding shell at the core of the nebulae in good correspondence with HST images. A full detailed description of this work will be published elsewhere.

\section{References}

Loṕez, J.A., 1997, IAU Symp. 180 on Planetary Nebulae, eds. H.J. Habing \& H. J. G. L. M. Lamers, pp 197 - 203.

Meaburn J., Blundell B., Carling R., Gregory, D.E., Keir, D.E., \& Wynne, C.G., 1984, MNRAS, 210, 463 

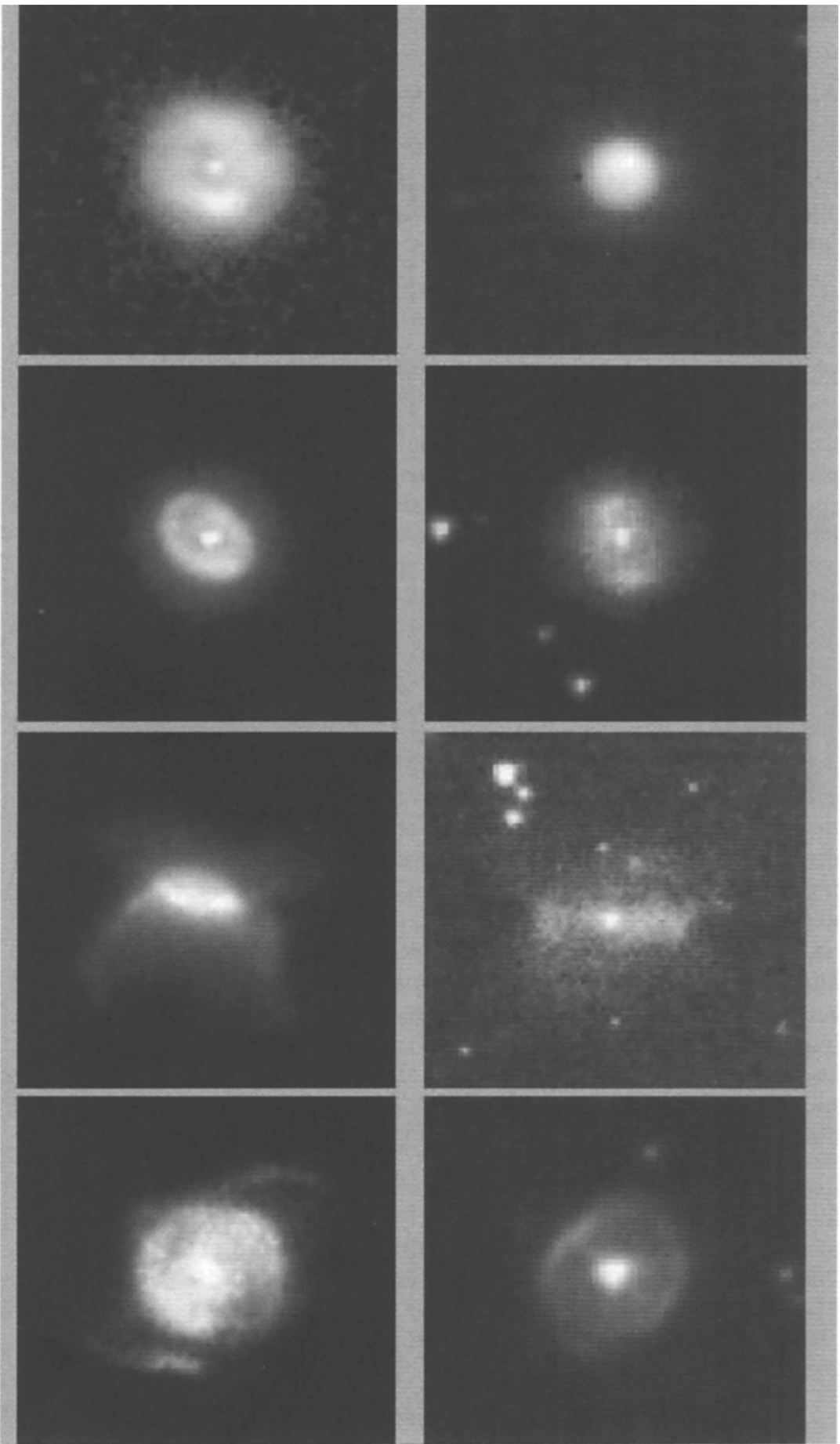

Examples of Magellanic Clouds planetary nebulae. Images courtesy of L Stanghellini. 